\title{
Optimization Models for Semiconductor Dopant Profiling
}

\author{
Martin Burger ${ }^{1}$, Michael Hinze ${ }^{2}$, and Rene Pinnau ${ }^{3}$ \\ 1 Institut für Industriemathematik, Johannes Kepler Universität, Altenbergerstr. \\ 69, A 4040 Linz, Austria martin.burger@jku.at \\ 2 Institut für Numerische Mathematik, Technische Universität Dresden, \\ Willersbau, C318, D-01062 Dresden, Germany hinze@math.tu-dresden.de \\ 3 Fachbereich Mathematik, Technische Universität Kaiserslautern, Erwin \\ Schrödinger Str., D-67663 Kaiserslautern, Germany \\ pinnau@mathematik. uni-kl.de
}

\section{Introduction}

Design of semiconductor devices is an important and challenging task in modern microelectronics, which is more and more carried out via mathematical optimization with models for the device behavior. The design variable (and correspondingly the unknown in the associated optimization problems) is the device doping profile, which describes the (charge) density of ion impurities in the device and is therefore modeled as a spatially inhomogeneous function. The optimization goals are usually related to the device characteristics, in particular to outflow currents on some contacts. This is also the typical setup we shall confine ourselves to in this paper, namely to (approximately) achieve a certain goal related the outflow current on a contact (e.g. a maximization or just an increase of the current), ideally with minimal change of the doping profile to some given reference state.

In order to solve such optimal design problems it is important to find suitable models of objective functionals to be minimized, so that a reasonable compromise between conflicting design goals (e.g. maximizing current and keeping the doping profile close to the reference state) can be achieved. We shall study and compare two different models that have been proposed for the optimization (and used for numerical solutions, cf. [HP02a, HP02b, BP03, HP05, HP06]). In any of the models, the weighting of the different goals leads to some parameters in the objective functionals and we shall pay particular attention to the limiting behavior of minimizers with respect to these parameters.

We shall start with an overview of models for the simulation and in particular for the optimization of semiconductor devices, which we carry out in a rather general setup. Then we turn our attention to a simple model case, 
namely the unipolar drift-diffusion model, for which a very detailed analysis of the optimization models can be carried out. We shall verify some fundamental properties such as existence of minimizers and existence of Lagrange multipliers, before we analyze the regularity of minimizers and the quite challenging problem of existence. Moreover, we also investigate the asymptotic behavior of the minimizers for large and small parameters in the objective functionals. Finally, we discuss the numerical solution of the optimization problems for the particular case of drift-diffusion models, but allowing bipolarity and multiple dimensions, and give some computational results.

\section{Models for Optimal Dopant Profiling}

Macroscopic models for semiconductor devices are usually composed of two basic state variables, namely the electric potential $V$ and a set of densities $\rho$ (e.g. electron and hole densities), which satisfy a nonlinear system of the form

$$
\begin{aligned}
-\lambda^{2} \Delta V & =Q(\rho)+C \\
F(\rho, V) & =0 .
\end{aligned}
$$

Here $\lambda$ denotes a scaling parameter (called Debye length), $Q(\rho)$ is the total charge density generated by $\rho, C$ is the doping profile (modeled as a function of space) and $F$ symbolizes nonlinear differential equations for $\rho$ (which also include the electric potential $V$ ). All equations are to be solved in a domain $\Omega$ modeling the device geometry and with suitable boundary conditions, which we do not further discuss here. For an overview of device models and their asymptotic relations we refer to [JP01, MRS90].

The primary optimization goal can usually be modeled in a straightforward way as a functional of the densities and the voltage, i.e.,

$$
R(V, \rho) \rightarrow \min _{(V, \rho, C) \text { satisfying (1),(2) }} .
$$

The functional $R$ could e.g. be the negative current outflow on a contact (in order to maximize the current (cf. [PSSS98, St00, Stea98]) or the square of current minus a target current (cf. [HP02a, HP02b]).

As an example we consider the most frequently used case, namely the bipolar drift-diffusion model, where $\rho=(n, p)$ with $n$ being the electron and $p$ the hole density. In this case the charge density is simply $Q=p-n$ and the differential operators included in $F$ are given by

$$
F(n, p)=\left(\begin{array}{c}
\nabla \cdot\left(D_{n} \nabla n-\mu_{n} n \nabla V\right) \\
\nabla \cdot\left(D_{p} \nabla p+\mu_{p} p \nabla V\right)
\end{array}\right)
$$

The current flowing out over a contact $\Gamma \subset \partial \Omega$ is then given by

$$
I=\int_{\Gamma} J \cdot d \nu, \quad J=D_{n} \nabla n-\mu_{n} n \nabla V-D_{p} \nabla p-\mu_{p} p \nabla V,
$$


and a prototypical optimization problem would be to minimize $-I$ or $\left|I-I^{*}\right|^{2}$.

It turns out that an optimization problem of the form (3) is not well-posed, i.e., the existence of solutions and the robustness of the problem cannot be guaranteed. In order to achieve these goals, a second term has to be introduced to the objective functional. In [HP02a, HP02b] an optimization of the form

$$
G_{\alpha}(V, \rho, C):=R(V, \rho)+\alpha\left\|C-C^{*}\right\|^{2} \rightarrow \underset{(V, \rho, C) \text { satisfying (1),(2) }}{\min }
$$

has been proposed. Here $C^{*}$ is a given prior for the doping profile and $\alpha$ is a positive parameter. If the norm is chosen appropriately it can be shown that a minimizer of (4) exists for $\alpha>0$. Moreover, one can formulate first-order optimality conditions, as usual in optimization based on the Lagrangian

$L(V, \rho, C ; p, q)=G_{\alpha}(V, \rho, C)+\int_{\Omega}\left(\lambda^{2} \nabla V \cdot \nabla p-Q(\rho) p-C p\right) d x+\langle F(\rho, V), q\rangle$

having zero variations with respect to the primal variables $(V, \rho, C)$ and the dual variables $(p, q)$. The latter just yields the constraints $(1),(2)$, which have to be coupled with

$$
\begin{aligned}
& 0=\frac{\partial L}{\partial V}=\frac{\partial R}{\partial V}(V, \rho)-\lambda^{2} \Delta p+\frac{\partial F}{\partial V}(\rho, V)^{*} q \\
& 0=\frac{\partial L}{\partial \rho}=\frac{\partial R}{\partial \rho}(V, \rho)-Q^{\prime}(\rho) p+\frac{\partial F}{\partial \rho}(\rho, V)^{*} q \\
& 0=\frac{\partial L}{\partial C}=\alpha E^{*} E\left(C-C^{*}\right)-p .
\end{aligned}
$$

Here $A^{*}$ denotes the adjoint of an operator $A$, and $E$ is the embedding operator from the space used for $C$ (with norm $\|$.$\| as used in the functional G_{\alpha}$ ) into $L^{2}(\Omega)$. Hence, the optimality conditions yield a system of five strongly coupled nonlinear equations (1), (2), (5), (6), (12), which is solved by a minimizer of (4). Both the analysis and the computation of minimizers turn out to be challenging tasks, which we shall investigate in more detail for a very special device model in the next section.

As an alternative to (4) a different approach has been introduced in [BP03], which is motivated from the structure of the above optimality system. It turns out that the optimality system can be simplified partially if a variable change from the doping profile $C$ to the total charge density

$$
W:=Q(\rho)+C
$$

is performed. Obviously, if one minimizes with respect to $(V, \rho, W)$ then one can reconstruct $C$ uniquely from this formula. On the other hand, the Poisson equation simplifies to

$$
-\lambda^{2} \Delta V=W
$$

i.e., the densities $\rho$ do not appear any more and the coupling between $V$ and $\rho$ becomes one-directional only. Since one is using a novel design variable $W$ 
in this setup it seems natural to adjust the penalizing term to this fact, i.e., to minimize

$$
H_{\beta}(V, \rho, W):=R(V, \rho)+\beta\left\|W-W^{*}\right\|^{2} \rightarrow \underset{(V, \rho, W) \text { satisfying (9),(2) }}{\min } .
$$

The Lagrangian associated to (10) is given by

$$
L(V, \rho, W ; p, q)=H_{\beta}(V, \rho, C)+\int_{\Omega}\left(\lambda^{2} \nabla V \cdot \nabla p-W p\right) d x+\langle F(\rho, V), q\rangle,
$$

and the optimality conditions are given by (9),(2) together with

$$
\begin{aligned}
0 & =\frac{\partial L}{\partial V}=\frac{\partial R}{\partial V}(V, \rho)-\lambda^{2} \Delta p+\frac{\partial F}{\partial V}(\rho, V)^{*} q \\
0 & =\frac{\partial L}{\partial \rho}=\frac{\partial R}{\partial \rho}(V, \rho)+\frac{\partial F}{\partial \rho}(\rho, V)^{*} q \\
0 & =\frac{\partial L}{\partial C}=\beta E^{*} E\left(W-W^{*}\right)-p .
\end{aligned}
$$

The structure of the optimality system for (10) turns out to be more convenient than the one for (4). For given design variable $W$ one can subsequently solve (9) for $V,(2)$ for $\rho,(12)$ for $q$, and (13) for $p$. For the case of the driftdiffusion model as stated above, this can be realized by solving scalar linear differential equations only instead of nonlinear coupled systems. As a direct consequence, the analysis of the optimality systems and important properties such as the existence of Lagrange multipliers $p$ and $q$ are rather straightforward (see [BP03] for the drift-diffusion model). Moreover, this decoupling of the optimality system can be used to construct efficient numerical methods as we will discuss in Section 4.

\section{Optimization of Unipolar Diodes}

In the following we provide a detailed analysis for the optimization of the unipolar drift-diffusion model for diodes. In this situation a spatially onedimensional analysis can be carried out, with a single density (namely the electron density $n$ ). For convenience we shall use a scaled version of the model and a standard transformation to so-called Slotboom variables and consider the unknown $u \sim e^{-V} n$ (cf. e.g. [MRS90] for details on scaling and density variables for the drift-diffusion model).

Motivated by the above discussion we consider the optimization problems (note from the model below that the current $J=e^{V} u_{x}$ is spatially homogeneous, so that $I=J$ )

$$
G_{\alpha}(u, V, C):=\frac{1}{2} \int_{0}^{1}\left|e^{V} u_{x}-J^{*}\right|^{2} d x+\frac{\alpha}{2}\left\|C-C^{*}\right\|^{2}
$$


and

$$
H_{\beta}(u, V, C):=\frac{1}{2} \int_{0}^{1}\left|e^{V} u_{x}-J^{*}\right|^{2} d x+\frac{\beta}{2}\left\|V_{x x}-V_{x x}^{*}\right\|^{2}
$$

both of them subject to

$$
\begin{aligned}
\lambda^{2} V_{x x}-e^{V} u & =-C & & \text { in }(0,1) \\
\left(e^{V} u_{x}\right)_{x} & =0 & & \text { in }(0,1) \\
V & =V^{*} & & \text { in }\{0,1\} \\
V_{x x} & =V_{x x}^{*} & & \text { in }\{0,1\} \\
u & =u_{D} & & \text { in }\{0,1\}
\end{aligned}
$$

In order to keep the notation as unified as possible in this section we shall not use the variable $W$ but directly write the problems in terms of $V$ and its derivatives.

Our aim is to study the parametric behavior of these functionals with respect to the positive real parameters $\alpha$ and $\beta$, respectively. In particular we shall investigate the asymptotic behavior of the minimizers as the parameters tend to zero or infinity, respectively. In the latter case, it seems obvious that the design variables ( $C$ and $V$, respectively) converge to their priors, which we will prove with a rate of at least $\alpha^{-1 / 2}$ and $\beta^{-1 / 2}$. In the case of parameters tending to zero numerical experiments indicate that the current $J=e^{V} u_{x}$ tends to the desired current $J^{*}$, which we will prove in both cases. For the functional $H_{\beta}$, we shall even prove that this convergence arises with rate $\sqrt{\beta}$ as $\beta \rightarrow 0$.

\subsection{Optimization for Positive and Finite Parameters}

In this section we shall investigate the optimization problems for parameter values $\alpha$ and $\beta$ in the open interval $(0,+\infty)$, which provides some basic results for the later asymptotic analysis, but also a variety of interesting results that yield further insight with respect to the parametric behavior of the optimization problems.

\section{Minimization of $G_{\alpha}$}

We start with a discussion of basic properties of the optimization problem (14) subject to (16), which was originally introduced [HP02a, HP02b].

Theorem 1. Let $\alpha>0, J^{*} \in \mathbb{R}, C^{*} \in H^{1}([0,1])$, and

$$
\left\|C-C^{*}\right\|^{2}=\int_{0}^{1}\left(\left|C_{x}-C_{x}^{*}\right|^{2}+\left|C-C^{*}\right|^{2}\right) d x
$$

Moreover let $u^{*} \in H^{1}([0,1]), V^{*} \in H^{3}([0,1])$ satisfy 


$$
\begin{array}{rlrl}
\lambda^{2} V_{x x}^{*} & =e^{V^{*}} u^{*}-C^{*} \text { in }(0,1) \\
\left(e^{V^{*}} u_{x}^{*}\right)_{x} & = & 0 & \text { in }(0,1) \\
u^{*} & = & u_{D}^{*} & \text { in }\{0,1\} .
\end{array}
$$

Then there exists a solution

$$
(\bar{u}, \bar{V}, \bar{C}) \in H^{1}([0,1]) \times H^{2}([0,1]) \times H^{1}([0,1])
$$

of the optimization problem (14), (16).

Proof. The existence of a solution $(\bar{u}, \bar{V}, \bar{C}) \in H^{1}([0,1])^{3}$ follows from a more general result in $[\mathrm{HP} 02 \mathrm{~b}]$, the additional regularity $\bar{V} \in H^{2}([0,1])$ in this one-dimensional case follows from

$$
\bar{V}_{x x}=e^{\bar{V}} \bar{u}-\bar{C} \in L^{2}([0,1]) .
$$

Besides the existence of a solution, the KKT-system and existence of Lagrange multipliers are of particular interest.

Proposition 1. Under the conditions of Theorem 1, there exist Lagrange multipliers $(\bar{p}, \bar{q}) \in H_{0}^{1}([0,1])^{2}$ such that a stationary point $(\bar{u}, \bar{V}, \bar{C})$ of (14), (16) satisfies

$$
\begin{array}{rlr}
0 & =-\alpha\left(\bar{C}_{x x}-C_{x x}^{*}\right)+\alpha\left(\bar{C}-C^{*}\right)-\bar{p} & \text { in }(0,1) \\
0 & =\left(e^{\bar{V}} \bar{u}_{x}-J^{*}\right) e^{\bar{V}} \bar{u}_{x}-\lambda^{2} \bar{p}_{x x}+e^{\bar{V}} \overline{p u}+e^{\bar{V}} \bar{u}_{x} \bar{q}_{x} & \text { in }(0,1) \\
0 & =-\left(e^{\bar{V}}\left(e^{\bar{V}} \bar{u}_{x}-J^{*}\right)\right)_{x}+e^{\bar{V}} \bar{p}-\left(e^{\bar{V}} \bar{q}_{x}\right)_{x} & \text { in }(0,1) \\
\bar{p} & =0 & \text { in }\{0,1\} \\
\bar{q} & =0 & \text { in }\{0,1\} .
\end{array}
$$

Proof. See [HP02b].

In general, one cannot expect the uniqueness of the Lagrange multipliers defined (5)-(7). But for the unipolar diodes considered here, the Lagrange multipliers are unique.

Theorem 2. Under the conditions of Proposition 1, the Lagrange multipliers $(\bar{p}, \bar{q}) \in H_{0}^{1}([0,1])^{2}$ are unique.

Proof. We consider the homogeneous system

$$
\begin{aligned}
& 0=-\lambda^{2} \bar{p}_{x x}+e^{\bar{V}} \overline{p u}+e^{\bar{V}} \bar{u}_{x} \bar{q}_{x} \quad \text { in }(0,1) \\
& 0=\quad+e^{\bar{V}} \bar{p}-\left(e^{\bar{V}} \bar{q}_{x}\right)_{x} \quad \text { in }(0,1)
\end{aligned}
$$

with $\bar{p}=\bar{q}=0$ in $\{0,1\}$. The second equation can be written as 


$$
\bar{p}=\bar{V}_{x} \bar{q}_{x}+\bar{q}_{x x}
$$

and plugging this in the first equation we get (with $n=e^{\bar{V}} \bar{u}$ )

$$
-\lambda^{2} \bar{p}_{x x}+\left(n \bar{q}_{x}\right)_{x}=0 .
$$

Hence, $-\lambda^{2} \bar{p}_{x}+n \bar{q}_{x}=k$ is constant. Now, we introduce $\xi:=e^{\bar{V}} \bar{q}_{x}$ and start again from the second equation to get

$$
\xi_{x x}=\left(e^{\bar{V}} \bar{p}\right)_{x}=e^{\bar{V}} \bar{p}_{x}+e^{\bar{V}} \bar{V}_{x} \bar{p}_{x}=\frac{1}{\lambda^{2}}\left(n \xi-k e^{\bar{V}}\right)+\bar{V}_{x} \xi_{x}
$$

as an equation for $\xi$ supplemented with boundary data $\xi_{x}(0)=\xi_{x}(1)=0$. From the boundary data for $\bar{q}$ we deduce

$$
\int_{0}^{1} e^{-\bar{V}} \xi d x=0
$$

Let $\xi \leq \xi \leq \bar{\xi}$ be sharp bounds. Choose a point $x_{0} \in[0,1]$ such that $\xi\left(x_{0}\right)=\bar{\xi}$ and $\xi_{x}\left(x_{0}\right)=0$. Then we have

$$
\xi_{x x}=\frac{1}{\lambda^{2}}\left(n \bar{\xi}-k e^{\bar{V}}\right) \leq 0
$$

and hence

$$
\bar{\xi} \leq \max _{x} \frac{k e^{\bar{V}}}{n} .
$$

In analogy, one shows

$$
\underline{\xi} \geq \min _{x} \frac{k e^{\bar{V}}}{n} .
$$

We deduce that $\xi$ does not change its sign and thus $\int_{0}^{1} e^{-\bar{V}} \xi d x=0$ implies that $\xi \equiv 0$ and then $\bar{q} \equiv 0$ and $\bar{p} \equiv 0$. Hence, the homogeneous problem has only the trivial solution which implies the uniqueness of the Lagrange multipliers.

Another typical property of an objective functional like (14) is that nonsmooth features of the solution $\bar{C}$ correspond to those in the prior $C^{*}$, or, in other words, $\bar{C}-C^{*}$ is very smooth. For rather general semiconductor devices optimized with respect to the objective $G_{\alpha}$, this effect was discussed in a formal way in [HP02b]. In the case of a unipolar diode considered here, this statement can be made rigorous as follows:

Theorem 3. Under the conditions of Proposition 1, a doping profile $\bar{C}$ corresponding to a stationary point $(\bar{u}, \bar{V}, \bar{C}, \bar{p}, \bar{q})$ solving (16), (17) satisfies

$$
\bar{C}-C^{*} \in H^{6}([0,1]) \hookrightarrow C^{5}([0,1]) .
$$


Proof. First of all, due to Proposition 1 we have

$$
\left(\bar{C}-C^{*}\right)_{x x}=\left(\bar{C}-C^{*}\right)+\frac{1}{\alpha} \bar{p} \in L^{2}(\Omega),
$$

from which we may conclude that $\bar{C}-C^{*} \in H^{2}([0,1])$. Moreover, (17) implies (noticing that $e^{\bar{V}} \bar{u}_{x}$ is constant)

$$
\begin{array}{lll}
\bar{p}_{x x}=\lambda^{-2}\left[\left(e^{\bar{V}} \bar{u}_{x}-J^{*}\right) e^{\bar{V}} \bar{u}_{x}+e^{\bar{V}} \overline{p u}+e^{\bar{V}} \bar{u}_{x} \bar{q}_{x}\right] & \in L^{2}([0,1]), \\
\bar{q}_{x x}=\bar{p}-\bar{V}_{x}\left(e^{\bar{V}} \bar{u}_{x}-J^{*}\right) & \in L^{2}([0,1]),
\end{array}
$$

and thus, $\bar{p} \in H^{2}([0,1])$ and $\bar{q} \in H^{2}([0,1])$. Using thus result, we deduce from the first line in (17) that

$$
\begin{array}{ll}
\frac{\partial^{3}}{\partial x^{3}}\left(\bar{C}-C^{*}\right)=\left(\bar{C}-C^{*}\right)_{x}+\alpha^{-1} \bar{p}_{x} & \in L^{2}([0,1]), \\
\frac{\partial^{4}}{\partial x^{4}}\left(\bar{C}-C^{*}\right)=\left(\bar{C}-C^{*}\right)_{x x}+\alpha^{-1} \bar{p}_{x x} & \in L^{2}([0,1]),
\end{array}
$$

i.e., $\bar{C}-C^{*} \in H^{4}([0,1])$.

By a further iteration of this process we obtain that

$$
\begin{aligned}
& \frac{\partial^{j} \bar{p}}{\partial x^{j}} \in L^{2}([0,1]), \\
& \frac{\partial^{j} \bar{q}}{\partial x^{j}} \in L^{2}([0,1]),
\end{aligned}
$$

for $j=3,4$, and consequently

$$
\frac{\partial^{j+2}}{\partial x^{j+2}}\left(\bar{C}-C^{*}\right)=\frac{\partial^{j}}{\partial x^{j}}\left(\bar{C}-C^{*}\right)+\alpha^{-1} \frac{\partial^{j} \bar{p}}{\partial x^{j}} \in L^{2}(\Omega),
$$

which implies $\bar{C}-C^{*} \in H^{6}([0,1]) \hookrightarrow C^{5}([0,1])$.

Note that the above result is obtained by a bootstrapping technique, from which one often derives $C^{\infty}$-regularity. The reason to stop at the the sixth derivative of $\bar{C}-C^{*}$ is that one cannot proceed further without assuming higher regularity than $C^{*} \in L^{2}(\Omega)$. If we want to have a bound on the seventh derivative of $\bar{C}-C^{*}$, we need bounds on the fifth derivative of $\bar{p}$, the third derivative of $\bar{V}$, and consequently, the first derivative of $\bar{C}$. But $\bar{C} \in H^{1}([0,1])$ is obtained only for

$$
C^{*}=\bar{C}-\left(\bar{C}-C^{*}\right) \in H^{1}([0,1])
$$




\section{Minimization of $\boldsymbol{H}_{\beta}$}

For the optimization problem (15), (16) we can prove similar results on the existence of minimizers and Lagrange multipliers as above:

Theorem 4. Let $\beta>0, J^{*} \in \mathbb{R}$, and $V^{*} \in H^{2}([0,1])$. Moreover, let $C^{*} \in$ $L^{2}([0,1])$ be defined by

$$
C^{*}=\lambda^{2} V_{x x}^{*}-e^{V^{*}} u^{*}
$$

where $u^{*}$ is the unique solution of

$$
-\left(e^{V^{*}} u_{x}^{*}\right)_{x}=0 \quad \text { in }(0,1),
$$

satisfying $u^{*}=u_{D}$ in $\{0,1\}$. Then, there exists a minimizer

$$
(\bar{u}, \bar{V}, \bar{C}) \in H^{1}([0,1]) \times H^{2}([0,1]) \times L^{2}([0,1])
$$

of (15) subject to (16).

Proof. See [BP03].

Proposition 2. Under the assumptions of Theorem 4, there exists a Lagrange multiplier $\bar{q} \in H_{0}^{1}([0,1])$ such that a stationary point $(\bar{u}, \bar{V})$ of (15), (16) satisfies

$$
\begin{aligned}
& \beta\left(\bar{V}_{x x}-V_{x x}^{*}\right)_{x x}+e^{\bar{V}} \bar{u}_{x}\left(e^{\bar{V}} \bar{u}_{x}-J^{*}\right)+e^{\bar{V}} \bar{u}_{x} \bar{q}_{x}=0 \quad \text { in }(0,1), \\
& -e^{\bar{V}} \bar{V}_{x}\left(e^{\bar{V}} \bar{u}_{x}-J^{*}\right)-\left(e^{\bar{V}} \bar{q}_{x}\right)_{x}=0 \quad \text { in }(0,1) .
\end{aligned}
$$

Proof. See [BP03].

The uniqueness of stationary points is a challenging problem, which seems to depend strongly on the problem setup. Here we consider a case related to numerical computations in [HP02b, BP03]), where the optimization was used to amplify an original given current $J^{0}$ by $50 \%$, i.e., $J^{*}=\frac{3}{2} J^{0}$. Since an optimization algorithm will be started with the reference state (and current $J=J^{0}$ ) and then increase the current one can expect that $J^{0} \leq J \leq J^{*}$ is the relevant situation for the current, and in this range uniqueness can be guaranteed:

Theorem 5. Let, in addition to the above conditions, $u_{D}(1)-u_{D}(0)>0$ and $J^{*}>0$. Then there is a unique stationary point $(\bar{u}, \bar{V}, \bar{q})$ of (15), (16), (20) among those functions satisfying satisfying $\frac{2}{3} J^{*} \leq J=e^{\bar{V}} \bar{u}_{x} \leq J^{*}$.

Proof. By integrating the second equation of (20) we obtain (with $J=e^{\bar{V}} \bar{u}_{x}$ )

$$
\bar{q}_{x}=J^{*}-J+c e^{-\bar{V}}
$$

for a constant $c$, which can be determined after integration from 0 to 1 as 


$$
c=\frac{J-J^{*}}{\int_{0}^{1} e^{-\bar{V}} d x}=\frac{J}{u_{D}(1)-u_{D}(0)}\left(J-J^{*}\right) .
$$

After plugging this into the first equation of (20), we end up with the equation

$$
\beta\left(\bar{V}_{x x}-V_{x x}^{*}\right)_{x x}+J c e^{-\bar{V}}=0 .
$$

The linearization of this equation is given by

$$
\beta \psi_{x x x x}-J c e^{-\bar{V}} \psi+\left(J^{\prime} c+J c^{\prime}\right) e^{-\bar{V}}=0,
$$

where $J^{\prime}$ and $c^{\prime}$ denote the derivatives of the functionals $J$ and $c$ with respect to $V$ in direction $\psi$, i.e.,

$$
J^{\prime}=\frac{u_{D}(1)-u_{D}(0)}{\left(\int_{0}^{1} e^{-\bar{V}} d x\right)^{2}} \int_{0}^{1} e^{-\bar{V}} \psi d x, \quad c^{\prime}=\frac{J^{\prime}\left(J-J^{*}\right)+J J^{\prime}}{u_{D}(1)-u_{D}(0)} .
$$

Note that for $0 \leq \frac{2}{3} J^{*} \leq J=e^{\bar{V}} \bar{u}_{x} \leq J^{*}$ we have

$$
J^{\prime} c+J c^{\prime}=J^{\prime} J \frac{3 J-2 J^{*}}{u_{D}(1)-u_{D}(0)} \geq 0, \quad c=\frac{J}{u_{D}(1)-u_{D}(0)}\left(J-J^{*}\right) \leq 0 .
$$

After multiplying (21) with $\psi$ and integration we obtain

$$
\int_{0}^{1}\left(\beta\left|\psi_{x x}\right|^{2}-J c e^{-\bar{V}} \psi^{2}\right) d x+J \frac{3 J-2 J^{*}}{u_{D}(1)-u_{D}(0)}\left(\int_{0}^{1} e^{-\bar{V}} \psi d x\right)^{2}=0 .
$$

Taking into account the signs of all the terms, this implies that $\psi=0$. Hence, the linearized problem has only the trivial solution, which implies the uniqueness of $\bar{V}$, and consequently of $\bar{u}$ and $\bar{q}$.

As for the minimization of $R_{\alpha}$, we can also derive a regularity result for the design variable. Since in this case, the real design variable is $\bar{V}-V^{*}$ it should not surprise that high regularity for this function can be obtained:

Theorem 6. Under the assumptions of Theorem 4, a stationary point $(\bar{u}, \bar{V}, \bar{V})$ solving (16), (20) satisfies

$$
\begin{aligned}
& \bar{V}-V^{*} \in H^{6}([0,1]) \hookrightarrow C^{5}([0,1]), \\
& \bar{C}-C^{*} \in H^{2}([0,1]) \hookrightarrow C^{1}([0,1]) .
\end{aligned}
$$

Proof. First of all, the function $w=\bar{V}-V_{x x}^{*}$ satisfies the Poisson equation

$$
-w_{x} x=\beta^{-1}\left(e^{\bar{V}} \bar{u}_{x}\left(e^{\bar{V}} \bar{u}_{x}-J^{*}\right)+e^{\bar{V}} \bar{u}_{x} \bar{q}_{x}\right) \in L^{2}([0,1]),
$$

with homogeneous boundary data, and by standard elliptic regularity we may conclude that $w \in H^{2}([0,1])$. Taking into account that $e^{\bar{V}} \bar{u}_{x}$ is constant, we obtain that 
for $j \geq 0$.

$$
\frac{\partial^{j+2} w}{\partial x^{j+2}}=-e^{\bar{V}} \bar{u}_{x} \frac{\partial^{j+1} \bar{q}}{\partial x^{j+1}},
$$

Due to (20) we have

$$
\begin{array}{ll}
\frac{\partial^{2} \bar{q}}{\partial x^{2}}=-\bar{V}_{x} \bar{q}_{x}-\bar{V}_{x}\left(e^{\bar{V}_{\bar{u}}}-J^{*}\right) & \in L^{2}([0,1]), \\
\frac{\partial^{3} \bar{q}}{\partial x^{3}}=-\bar{V}_{x} \bar{q}_{x x}-\bar{V}_{x x} \bar{q}_{x}-\bar{V}_{x x}\left(e^{\bar{V}} \bar{u}_{x}-J^{*}\right) & \in L^{2}([0,1]),
\end{array}
$$

and as a consequence we obtain that $\bar{q} \in H^{3}([0,1]), w \in H^{4}([0,1])$, and hence, $\bar{V}-V^{*} \in H^{6}([0,1]) \hookrightarrow C^{5}([0,1])$.

Finally, from the Poisson equation we deduce

$$
\frac{\partial^{j}}{\partial x^{j}}\left(\bar{C}-C^{*}\right)=\frac{\partial^{j}}{\partial x^{j}}\left(\lambda^{2} W-e^{\bar{V}} \bar{u}+e^{V^{*}} u^{*}\right) \in L^{2}([0,1]) .
$$

for $j=0,1,2$, and thus, $\left(\bar{C}-C^{*}\right) \in H^{2}([0,1])$.

Note that in this case we obtain $H^{6}$-regularity of the design variable also with a rather weak penalization term on $W$ in the $L^{2}$-norm. If we would use the $H^{1}$-norm of $W$ for the penalty instead, this would even imply $H^{8}$ regularity of $\bar{V}-V^{*}$. For the change in the doping profile $\bar{C}-C^{*}$ we cannot obtain higher regularity than $H^{2}$, since this would enforce the existence of higher than second derivatives of $\bar{V}$ and $V^{*}$, or, due to the Poisson equation, the existence of derivatives for $\bar{C}$ and $C^{*}$, which we do not assume here.

\subsection{Asymptotic Behavior}

In the following we investigate the two different limits for the parameters $\alpha$ and $\beta$, namely convergence to zero and infinity, respectively

\section{Large Parameters}

The limit of the parameter $\alpha$ and $\beta$ tending to infinity is the easier case, it seems obvious that the solutions of the optimization problems (14) or (15) subject to $(16)$ converge to the prior $\left(u^{*}, V^{*}, C^{*}\right)$. From the structure of the objective we can prove that this convergence happens with rate $\mathcal{O}\left(\alpha^{-1 / 2}\right)$ and $\mathcal{O}\left(\beta^{-1 / 2}\right)$, respectively.

Theorem 7. Let $\alpha>0$ and $J^{*} \in \mathbb{R}$. Moreover, denote by $\left(u^{\alpha}, V^{\alpha}, C^{\alpha}\right)$ the unique solution of the optimization problem (14), (15) for fixed $\alpha$. Then there exists a positive real constant $M$ such that

$$
\left\|V^{\alpha}-V^{*}\right\|_{H^{3}}+\left\|u^{\alpha}-u^{*}\right\|_{H^{1}}+\left\|C^{\alpha}-C^{*}\right\|_{H^{1}} \leq \frac{M}{\sqrt{\alpha}}
$$

for $\alpha$ sufficiently large. In particular, $\left(u^{\alpha}, V^{\alpha}, C^{\alpha}\right) \rightarrow\left(u^{*}, V^{*}, C^{*}\right)$. 
Proof. From(14) we immediately obtain

$$
\left\|e^{V^{\alpha}} u_{x}^{\alpha}-J^{*}\right\|_{L^{2}}^{2}+\alpha\left\|C^{\alpha}-C^{*}\right\|_{H^{1}}^{2} \leq\left\|e^{V^{*}} u_{x}^{*}-J^{*}\right\|^{2}
$$

and thus,

$$
\left\|C^{\alpha}-C^{*}\right\|_{H^{1}} \leq \frac{M_{0}}{\sqrt{\alpha}}
$$

From the well-posedness of the drift-diffusion model for unipolar diodes [MRS90, GS92] we may conclude that

$$
\left\|V^{\alpha}-V^{*}\right\|_{H^{1}}^{2}+\left\|u^{\alpha}-u^{*}\right\| \leq \gamma\left\|C^{\alpha}-C^{*}\right\|_{H^{1}} \leq \gamma \frac{M_{0}}{\sqrt{\alpha}},
$$

for some constant $\gamma>0$ independent of $\alpha$. Finally, from the Poisson equation we deduce with the above $H^{1}$-estimates that

$\frac{\partial^{j+2}}{\partial x^{j+2}}\left(V^{\alpha}-V^{*}\right)=\lambda^{-2} \frac{\partial^{j}}{\partial x^{j}}\left(e^{V^{\alpha}}\left(u^{\alpha}-u^{*}\right)+\left(e^{V^{\alpha}}-e^{V^{*}}\right) u^{*}+\left(C^{\alpha}-C^{*}\right)\right) \in L^{2}([0,1])$,

for $j=0,1$, and from the above estimates on the $H^{1}$-norms we can also conclude that

$$
\left\|V^{\alpha}-V^{*}\right\|_{H^{3}} \leq \frac{M_{1}}{\sqrt{\alpha}}
$$

for some constant $M_{1}$, which completes the proof.

An analogous result holds for the limit $\beta \rightarrow \infty$ in the minimization of $H_{\beta}$ :

Theorem 8. Let $\beta>0$ be sufficiently large, $J^{*} \in \mathbb{R}$, and denote by $\left(u^{\beta}, V^{\beta}, C^{\beta}\right)$ the unique minimizer of (15), (16) for fixed $\beta$. Then there exists a constant $M>0$ such that

$$
\left\|V^{\beta}-V^{*}\right\|_{H^{2}}+\left\|u^{\beta}-u^{*}\right\|_{H^{1}}+\left\|C^{\beta}-C^{*}\right\|_{L^{2}} \leq \frac{M}{\sqrt{\beta}} .
$$

Proof. As in the proof of Theorem 7 we may deduce that

$$
\left\|e^{V^{\beta}} u_{x}^{\beta}-J^{*}\right\|_{L^{2}}^{2}+\beta\left\|V_{x x}^{\beta}-V_{x x}^{*}\right\|_{L^{2}}^{2} \leq\left\|e^{V^{*}} u_{x}^{*}-J^{*}\right\|^{2}
$$

which implies by standard reasoning that

$$
\left\|V^{\beta}-V^{*}\right\|_{H^{2}} \leq \frac{M_{0}}{\sqrt{\beta}}
$$

for some constant $M_{0}$. Since

$$
\int_{0}^{1} e^{V^{*}}\left|u_{x}^{\beta}-u^{*}\right|^{2} d x=\int_{0}^{1}\left(e^{V^{*}}-e^{V^{\beta}}\right) u_{x}^{\beta}\left(u_{x}^{\beta}-u^{*}\right) d x
$$

we may conclude from the Cauchy-Schwarz inequality and the uniform boundedness of $u_{x}^{\beta}$ that 


$$
\left\|u^{\beta}-u^{*}\right\|_{H^{1}} \leq \gamma\left\|V^{\beta}-V^{*}\right\|_{H^{2}} \leq \gamma \frac{M_{0}}{\sqrt{\beta}},
$$

for some constant $\gamma$. Finally, the estimate for $\left\|C^{\beta}-C^{*}\right\|_{L^{2}}$ follows from

$$
C^{\beta}-C^{*}=\lambda^{2}\left(V_{x x}^{\beta}-V_{x x}^{*}\right)-e^{V^{*}}\left(u^{\beta}-u^{*}\right)+\left(e^{V^{*}}-e^{V^{\beta}}\right) u^{\beta}
$$

and a standard Lipschitz estimate.

\section{Small Parameters}

We shall now turn our attention to the limit case of $\alpha \rightarrow 0$ and $\beta \rightarrow 0$, respectively, where we may expect that $e^{\bar{V}} \bar{u} \rightarrow J^{*}$. In order to obtain further insight, we directly start with the limit problem, which is the same for $\alpha=0$ and $\beta=0$. One might expect that the limit is determined by the equation

$$
e^{V^{0}} u_{x}^{0}=J^{*}
$$

subject to (16), for which one has to expect an infinite number of solutions. Taking into account the fact that we actually want to compute an optimal design that is as close as possible to the original one, a more suitable limit problem for (14), (16) is given by

$$
\frac{1}{2}\left\|C-C^{*}\right\|_{H^{1}}^{2} \rightarrow \min _{(u, V, C)},
$$

subject to (16), (22). In an analogous way we define a limit problem for (15), (16) as

$$
\frac{1}{2}\left\|V_{x x}-V_{x x}^{*}\right\|_{L^{2}}^{2} \rightarrow \min _{(u, V, C)},
$$

subject to $(16),(22)$.

We first make sure that the feasible set of these problems defined by (16), (22) is nonempty:

Lemma 1. Let $V \in H^{2}([0,1])$ satisfy

$$
\int_{0}^{1} e^{-V} d x=\frac{u_{D}(1)-u_{D}(1)}{J^{*}} \neq 0
$$

as well as the boundary conditions

$$
V=V^{*}, \quad V_{x x}=V_{x x}^{*} \quad \text { in }\{0,1\} .
$$

Then, there exists $u \in H^{1}([0,1])$ and $C \in L^{2}([0,1])$ such that (16) and $(22)$ hold. Moreover, $C \in H^{1}([0,1])$ if $V \in H^{3}([0,1])$ and $C^{*} \in H^{1}([0,1])$. 
Proof. For $V$ satisfying the above conditions, there exists a unique weak solution $u \in H^{1}([0,1])$ of the elliptic equation

$$
\left(e^{V} u_{x}\right)_{x}=0 \quad \text { in }(0,1)
$$

with boundary values $u=u_{D}$ in $\{0,1\}$. Moreover, there exists a unique doping profile $C \in L^{2}([0,1])$ defined via

$$
C=C^{*}-\lambda^{2}\left(V_{x x}-V_{x x}^{*}\right)+e^{V} u-e^{V^{*}} u^{*} .
$$

Since the derivative of the last two terms involves only first derivatives of the variables, which exist anyway under the above assumptions, we obtain $C \in H^{1}([0,1])$ if $V \in H^{3}([0,1])$ and $C^{*} \in H^{1}([0,1])$.

Now let $J:=e^{V} u_{x}$, which is a constant in $(0,1)$. Then, from (25) we deduce that

$$
\begin{aligned}
u_{D}(1)-u_{D}(0) & =\int_{0}^{1} u_{x} d x=J \int_{0}^{1} e^{-V} d x \\
& =\frac{J}{J^{*}}\left(u_{D}(1)-u_{D}(0)\right),
\end{aligned}
$$

and hence, $J=J^{*}$. From the above construction we observe that (16) and (22) hold, which completes the proof.

This result indicates that at least for a reasonable range of parameters, it should be possible to find a solution of (16), (22). Indeed, we shall show that this range is determined by the signs of $u_{D}(1)-u_{D}(0)$ only.

Theorem 9. Let $u_{D}(1)-u_{D}(0)>0(<0$, respectively). Then the feasible set determined by (16) and (22) is nonempty for each $J^{*}>0(<0)$.

Proof. Due to Lemma 1, it suffices to find a $V \in H^{3}([0,1])$ satisfying the boundary conditions and (25). Since the right-hand side in this relation does not change when we change both the sign of $\left(u_{D}(1)-u_{D}(0)\right)$ and $J^{*}$, we may restrict our attention to the case of both being positive. Let $\tilde{V}$ be a function in $H^{3}([0,1])$ that satisfies the boundary conditions in (16), e.g., a polynomial of order three. Moreover, let $W \in H^{3}([0,1])$ be a nonnegative function with compact support and $W \equiv 1$ for $x \in\left[\frac{1}{4}, \frac{3}{4}\right]$. We define $V_{t}=\tilde{V}+t W$ for $t \in \mathbb{R}$, and

$$
I(t):=\int_{0}^{1} e^{-V_{t}} d x
$$

It is easy to see that $I$ is a continuous function and

$$
\lim _{t \rightarrow-\infty} I(t)=0, \quad \lim _{t \rightarrow+\infty} I(t)=\infty,
$$

and hence $\mathbb{R}^{+}$is included in the range of the function $I$. Consequently, we can obtain a feasible point of (16), (22) for each $J^{*} \in \mathbb{R}^{+}$(by choosing $t$ such that $\left.I(t)=J^{*}\right)$. 
Due to the above result we shall assume in the following that $\left(u_{D}(1)-\right.$ $\left.u_{D}(0)\right) J^{*}>0$, such that there exists a feasible point of the limit problem.

We shall consider the limit problem of minimizing

$$
\frac{1}{2}\left\|V_{x x}-V_{x x}^{*}\right\|_{L^{2}}^{2} \rightarrow \min _{V \in H^{2}([0,1])} .
$$

subject to (25) and the boundary conditions in (16). Since the feasible set is nonempty and weakly closed under the above conditions, we may conclude the existence of a minimizer. Due to standard first-order optimality, the minimizer $\bar{V}$ satisfies

$$
\int_{0}^{1}\left(\bar{V}_{x x}-V_{x x}^{*}\right) W_{x x} d x=0
$$

for all $W \in H^{2}([0,1])$ with homogeneous boundary values and

$$
\int_{0}^{1} e^{-\bar{V}} W d x=0 .
$$

For arbitrary $W$ we can find a decomposition of the form $W=\mu W_{0}+(W-$ $\left.\mu W_{0}\right)$ for a fixed element $W_{0}$ satisfying

$$
\int_{0}^{1} e^{-\bar{V}} W_{0} d x \neq 0
$$

and

$$
\mu=\frac{\int_{0}^{1} e^{-\bar{V}} W d x}{\int_{0}^{1} e^{-\bar{V}} W_{0} d x}
$$

Thus, we have

$$
\int_{0}^{1}\left(\bar{V}_{x x}-V_{x x}^{*}\right) W_{x x} d x-p_{0} \int_{0}^{1} e^{\bar{V}} W d x=0,
$$

for arbitrary $W \in H^{2}([0,1])$ with homogeneous boundary values, and the Lagrange multiplier

$$
p_{0}=\frac{\int_{0}^{1} e^{-\bar{V}} W d x}{\int_{0}^{1}\left(\bar{V}_{x x}-V_{x x}^{*}\right)\left(W_{0}\right)_{x x} d x} .
$$

That means, $\bar{V}$ is a weak solution of the fourth-order equation

$$
\left(V_{x x}-V_{x x}^{*}\right)_{x x}=p_{0} e^{-V} .
$$

As a consequence, we can derive the following result on the existence of Lagrange multipliers for the limit problem: 
Proposition 3. Let $\left(u_{D}(1)-u_{D}(0)\right) J^{*}>0$, and let $(\bar{V}, \bar{u}, \bar{C})$ be a minimizer of (26) subject to (16), (22). Then there exist Lagrangian variables

$$
(\bar{p}, \bar{q}, \bar{r}) \in H_{0}^{1}([0,1]) \times H^{1}([0,1]) \times L^{2}([0,1]),
$$

such that

$$
\mathcal{L}^{\prime}(\bar{u}, \bar{V}, \bar{C} ; \bar{p}, \bar{q}, \bar{r})=0
$$

for the Lagrangian

$$
\begin{aligned}
\mathcal{L}(u, V, C ; p, q, r)= & \frac{1}{2}\left\|V_{x x}-V_{x x}^{*}\right\|_{L^{2}}^{2}+ \\
& \int_{0}^{1}\left(e^{V}\left(u_{x} p_{x}+u_{x} q\right)-J^{*} q+\lambda^{2} V_{x} r_{x} e^{V} u r-C r\right) d x .
\end{aligned}
$$

Moreover, $\bar{p}=\bar{r}=0$.

Proof. Let $\bar{q}=-\frac{p_{0}}{J^{*}} e^{-\bar{V}}$, with $p_{0}$ and $\bar{V}$ as above, and let $\bar{p}=\bar{r}=0$. Then we obtain

$$
\begin{aligned}
\frac{\partial}{\partial u} \mathcal{L}(\bar{u}, \bar{V}, \bar{C} ; \bar{p}, \bar{q}, \bar{r}) & =-\left(e^{\bar{V}} \bar{p}_{x}+e^{\bar{V}} \bar{q}\right)_{x}+e^{\bar{V}} \bar{r}=\left(\frac{p_{0}}{J^{*}}\right)_{x}=0 \\
\frac{\partial}{\partial V} \mathcal{L}(\bar{u}, \bar{V}, \bar{C} ; \bar{p}, \bar{q}, \bar{r}) & =\left(\bar{V}_{x x}-V_{x x}^{*}\right)_{x x}+e^{\bar{V}} \bar{u}_{x} \bar{q}+e^{\bar{V}} \bar{u}_{x} \bar{p}_{x}-\lambda^{2} \bar{r}_{x x}+e^{\bar{V}} \overline{u r} \\
& =\left(\bar{V}_{x x}-V_{x x}^{*}\right)_{x x}-p_{0} e^{-\bar{V}}=0 \\
\frac{\partial}{\partial C} \mathcal{L}(\bar{u}, \bar{V}, \bar{C} ; \bar{p}, \bar{q}, \bar{r}) & =-\bar{r}=0 .
\end{aligned}
$$

Since $(\bar{u}, \bar{V}, \bar{C})$ satisfies the constraints (16) and (22) the derivatives with respect, to the Lagrangian variables vanish, too, and thus, $\mathcal{L}^{\prime}(\bar{u}, \bar{V}, \bar{V} ; \bar{p}, \bar{q}, \bar{r})=$ 0.

The existence of Lagrange multipliers for the limit problem allows to derive a quantitative convergence result for $\beta \rightarrow 0$.

Theorem 10. Let $\left(\beta_{k}\right)$ be a sequence of positive numbers converging to zero, and let $\left(u_{k}, V_{k}, C_{k}\right)$ be a sequence of minimizers of (15), (16). Then there exists a subsequence converging to a minimizer $(\bar{u}, \bar{V}, \bar{V})$ of (26), (16). Moreover, each such subsequence (without restriction of generality $\left(u_{k}, V_{k}, C_{k}\right)$ itself) satisfies

$$
\left\|V_{k}-\bar{V}\right\|_{H^{2}}+\left\|u_{k}-\bar{u}\right\|_{H^{1}}+\left\|C_{k}-C\right\|_{L^{2}} \leq m \sqrt{\beta_{k}},
$$

for some constant $m \in \mathbb{R}^{+}$. Moreover, the fitting term satisfies

$$
\left\|e^{V_{k}}\left(u_{k}\right)_{x}-J^{*}\right\|_{L^{2}} \leq m_{0} \beta_{k}
$$

for some constant $m_{0} \in \mathbb{R}^{+}$. 
Proof. Due to the existence of Lagrangian variables $(\bar{p}, \bar{q}, \bar{r})$ we obtain that

$$
\mathcal{L}(\bar{u}, \bar{V}, \bar{C} ; \bar{p}, \bar{q}, \bar{r}) \leq \mathcal{L}\left(u_{k}, V_{k}, C_{k} ; \bar{p}, \bar{q}, \bar{r}\right)
$$

and because of $\bar{p}=\bar{r}=0$, this implies

$$
\begin{aligned}
\frac{1}{2}\left\|\bar{V}_{x x}-V_{x x}^{*}\right\|_{L^{2}}^{2} & \leq \frac{1}{2}\left\|\left(V_{k}\right)_{x x}-V_{x x}^{*}\right\|_{L^{2}}^{2}+\int_{0}^{1} \bar{q}\left(e^{V_{k}}\left(u_{k}\right)_{x}-J^{*}\right) d x \\
& \leq \frac{1}{2}\left\|\left(V_{k}\right)_{x x}-V_{x x}^{*}\right\|_{L^{2}}^{2}+\|\bar{q}\|_{L^{2}}\left\|e^{V_{k}}\left(u_{k}\right)_{x}-J^{*}\right\|_{L^{2}} .
\end{aligned}
$$

On the other hand, since $\left(V_{k}, u_{k}, C_{k}\right)$ is a minimizer of (15), (16) with $\beta=\beta_{k}$, we have

$$
\frac{1}{2}\left\|\left(V_{k}\right)_{x x}-V_{x x}^{*}\right\|_{L^{2}}^{2}+\frac{1}{\beta_{k}}\left\|e^{V_{k}}\left(u_{k}\right)_{x}-J^{*}\right\|_{L^{2}}^{2} \leq \frac{1}{2}\left\|\bar{V}_{x x}-V_{x x}^{*}\right\|_{L^{2}}^{2} .
$$

By combining these estimates, we may conclude that

$$
\left\|e^{V_{k}}\left(u_{k}\right)_{x}-J^{*}\right\|_{L^{2}} \leq \beta_{k}\|\bar{q}\|_{L^{2}}
$$

and subsequently

$$
\|\| \bar{V}_{x x}-V_{x x}^{*}\left\|_{L^{2}}^{2}-\right\|\left(V_{k}\right)_{x x}-V_{x x}^{*}\left\|_{L^{2}}^{2} \mid \leq 2 \beta_{k}\right\| \bar{q} \|_{L^{2}} .
$$

Thus,

$$
\begin{aligned}
\left\|\bar{V}_{x x}-\left(V_{k}\right)_{x x}\right\|_{L^{2}}^{2} & =\left\|\bar{V}_{x x}-V_{x x}^{*}\right\|_{L^{2}}^{2}-\left\|\left(V_{k}\right)_{x x}-V_{x x}^{*}\right\|_{L^{2}}^{2} \\
& \quad-2 \int_{0}^{1}\left(\bar{V}_{x x}-\left(V_{k}\right)_{x x}\right)\left(\bar{V}_{x x}-V_{x x}^{*}\right) d x \\
& \leq 2 \beta_{k}\|\bar{q}\|_{L^{2}}-2 \int_{0}^{1} e^{\bar{V}}\left(\bar{V}-V_{k}\right) \bar{u}_{x} \bar{q} d x \\
& =2 \beta_{k}\|\bar{q}\|_{L^{2}}+2 p_{0} \int_{0}^{1} e^{-\bar{V}}\left(\bar{V}-V_{k}\right) d x=2 \beta_{k}\|\bar{q}\|_{L^{2}},
\end{aligned}
$$

where we have used $\bar{q}=-\frac{p_{0}}{J^{*}} e^{-\bar{V}}$ and $J^{*}=e^{\bar{V}} \bar{u}_{x}$ in the last identity. From the first-order optimality for the limit problem the second term on the right-hand side vanishes and hence,

$$
\left\|\bar{V}_{x x}-\left(V_{k}\right)_{x x}\right\|_{L^{2}} \leq \sqrt{2\|\bar{q}\|_{L^{2}} \beta_{k}} .
$$

The estimate (27) follows from Poincaré inequalities and standard stability estimates for the equations in (16). 


\section{Numerical Solution of the Optimization Problems}

Numerical algorithms for the solution of (4) are either based on a steepest descent approach or on the solution of the first order optimality condition given by (1), (2) and (5)-(7) via Newton's method (cf. [HP05, HP06]). The same approaches might be used for the minimization problem (10), but due to the special structure of the first order optimality condition one might use a variant of the well-known Gummel iteration instead (cf. [BP03]). All three approaches are discussed in the following and numerical examples for the optimal dopant profiling of an unsymmetric $n-p$-diode are presented, where the underlying model equations are given by the stationary bipolar drift diffusion equations without generation-recombination terms (cf. [MRS90]). Stated on the interval $\Omega=(0,1)$ the scaled bipolar model reads

$$
\begin{gathered}
J_{n}=\left(n_{x}-n V_{x}\right), \quad J_{p}=-\left(p_{x}+p V_{x}\right), \\
\partial_{x} J_{n}=0, \quad \partial_{x} J_{p}=0, \\
-\lambda^{2} V_{x x}=C-n+p .
\end{gathered}
$$

Here, we only considered regimes in which we can assume the Einstein relations

$$
D_{n}=U_{T} \mu_{n}, \quad D_{p}=U_{T} \mu_{p},
$$

where $U_{T}=k_{B} T / q$ is the thermal voltage of the device and $T$ denotes its temperature and $k_{B}$ the Boltzmann constant.

This system is supplemented with the following boundary conditions:

$$
n=n_{D}, \quad p=p_{D}, \quad V=V_{D} \quad \text { in }\{0,1\},
$$

where $n_{D}, p_{D}, V_{D}$ are the $H^{1}(0,1)$-extensions of

$$
\begin{aligned}
& n_{D}=\frac{C+\sqrt{C^{2}+4 \delta^{4}}}{2}, \quad p_{D}=\frac{-C+\sqrt{C^{2}+4 \delta^{4}}}{2}, \\
& V_{D}=-\log \left(\frac{n_{D}}{\delta^{2}}\right)+U,
\end{aligned}
$$

where $\delta^{2}=n_{i} / C_{m}$ denotes the scaled intrinsic density.

\subsection{Gradient-based Methods}

The formulation of a gradient-based steepest descent method for the optimization problem (4) relies on the introduction of the so-called reduced cost functional $\hat{G}_{\alpha}(C):=G_{\alpha}(V(C), \rho(C), C)$, where $(V(C), \rho(C))$ is the solution of $(1),(2)$. Clearly, this is only possible if the nonlinear system admits a unique solution. For the unipolar diodes in one spatial dimension, as discussed in Section 3, this holds due to a result in [GS92] for the stationary drift diffusion model. In general, we can expect the uniqueness only near to the thermal equilibrium state, i.e. for small applied biasing voltages.

The gradient algorithm for the reduced cost functional $\hat{G}_{\alpha}$ reads (cf. [HP05]): 
1. Choose an admissible $C_{0}$.

2. For $k=1,2, \ldots$ compute

$$
C_{k}=C_{k-1}-\delta_{k} \hat{G}_{\alpha}^{\prime}\left(C_{k-1}\right) .
$$

The step-size $\delta_{k}$ is computed by an exact one-dimensional line-search

$$
\delta_{k}=\operatorname{argmin}_{\delta} \hat{G}_{\alpha}\left(C_{k-1}-\delta \hat{G}_{\alpha}^{\prime}\left(C_{k-1}\right)\right) .
$$

and the algorithm is terminated if the relative error $\left\|\hat{G}_{\alpha}^{\prime}\left(C_{k}\right)\right\| /\left\|\hat{G}_{\alpha}^{\prime}\left(C_{0}\right)\right\|$ is less than a specified error tolerance.

The evaluation of $\hat{G}_{\alpha}^{\prime}$ requires the solution of the nonlinear state system (1), (2) as well as a solution of the linear adjoint system (5), (6). Hence, each gradient step yields a feasible point. Compared with black-box optimization we have the advantage that this algorithm is independent of the number of discrete design variables given by a suitable discretization of $C$.

Nevertheless, one has to admit that the main part of the numerical work is hidden in the line search, since each evaluation of the reduced cost functional also requires the solution of the nonlinear state system. Instead of the exact line search one could use here e.g. Armijo's rule, which will still give sufficient decrease of the cost functional to ensure convergence.

To get an impression of the performance of the algorithm we present in Figure 1 the optimized doping profiles for an unsymmetric $n-p-$ diode where the observation is given by

$$
R\left(\left.J_{n} \cdot \nu\right|_{\Gamma},\left.J_{p} \cdot \nu\right|_{\Gamma}\right)=\frac{1}{2}\left|\int_{\Gamma} J_{n} \cdot \nu d s-I_{n}^{*}\right|^{2}+\frac{1}{2}\left|\int_{\Gamma} J_{p} \cdot \nu d s-I_{p}^{*}\right|^{2}
$$

and the state system is given by the standard drift diffusion model without generation-recombination terms. This allows to adjust the electron and hole current separately. Especially, we present the optimized doping profiles for different choices of $I_{n}^{*}, I_{p}^{*}$, i.e. we are seeking an amplification of either the hole current $\left(I_{n}^{*}=J_{n}^{*}, I_{p}^{*}=1.5 \cdot J_{p}^{*}\right)$ or of the electron current $\left(I_{n}^{*}=1.5 \cdot J_{n}^{*}, I_{p}^{*}=\right.$ $\left.J_{p}^{*}\right)$ or of both of them $\left(I_{n}^{*}=1.5 \cdot J_{n}^{*}, I_{p}^{*}=1.5 \cdot J_{p}^{*}\right)$ by $50 \%$. To get an impression of the overall performance of the method we also have to consider the nonlinear solves needed for the exact one-dimensional line-search. These are presented in Figure 2 and one realizes that this is indeed the numerically most expensive part.

\subsection{Newton Methods}

Newton-type methods for the solution of the optimization problem (4) are mainly based on the direct solution of the first order optimality condition given by (1), (2) and (5)-(7) or, with help of the Lagrangian,

$$
L^{\prime}(V, \rho, C ; p, q)=0 .
$$



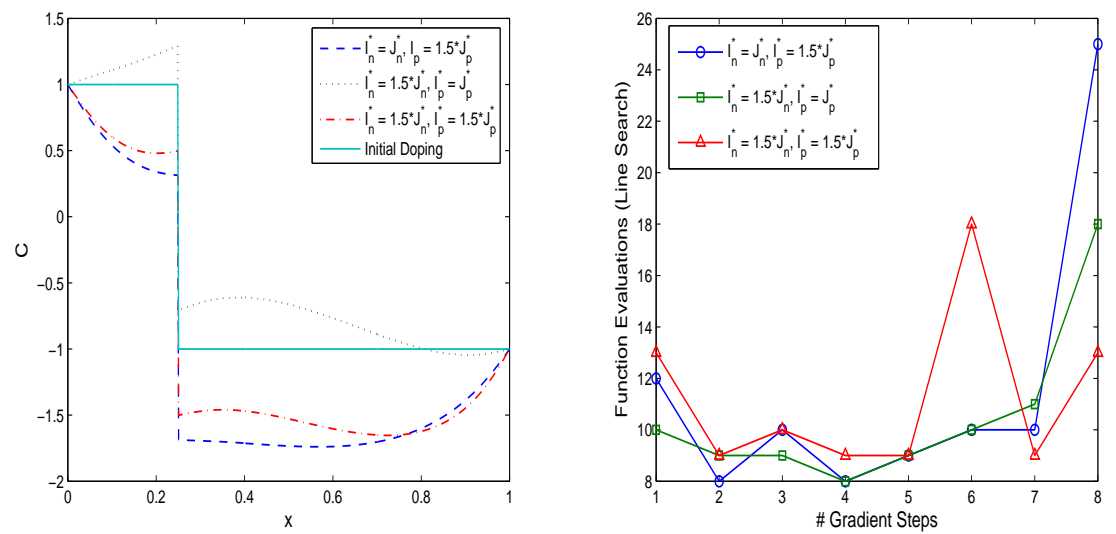

Fig. 1. Optimized doping profiles for dif- Fig. 2. Number of evaluations of $\hat{G}_{\alpha}$ per ferent design goals gradient step.

This coupled nonlinear system is then solved iteratively using its Jacobian $L^{\prime \prime}(V, \rho, V ; p, q)$, which formally reads

$$
\begin{aligned}
& L^{\prime \prime}(V, \rho, C ; p, q)= \\
& {\left[\begin{array}{ccc}
G_{\alpha, x x}(x, C)+\left\langle e_{x x}(x, C)(\cdot, \cdot),(p, q)\right\rangle & 0 & e_{x}(x, C)^{*} \\
0 & G_{\alpha, C C}(x, C) & e_{C}(x, C)^{*} \\
e_{x}(x, C) & e_{C}(x, C) & 0
\end{array}\right],}
\end{aligned}
$$

where we used for brevity the notation $x=(V, \rho)$ and the operator $e$ is defined via $e(x, C)=\left(F(v, \rho),-\lambda^{2} \Delta V-Q(\rho)-C\right)$. Further, for notational convenience we define the state-control pair $y \stackrel{\text { def }}{=}(x, C)$.

If the state system admits a unique solution, we can again introduce the reduced cost functional $\hat{G}_{\alpha}(C) \stackrel{\text { def }}{=} G_{\alpha}(x(C), C)$, where $x(C)$ is determined by $e(x(C), C)=0$. The derivative of the reduced cost functional is given by

$$
\hat{G}_{\alpha}^{\prime}(C)=G_{\alpha, C}(y(C))+e_{C}^{*}(y(C))(p, q),
$$

where $(p, q)$ solves the adjoint equations $(5),(6)$. We recall that unique solvability of $e(x, C)=0$ is ensured for devices operated near thermal equilibrium, i.e. for devices with small applied biasing voltages or for the onedimensional unipolar diode.

Now we derive Newton's method for the solution of

$$
\hat{G}_{\alpha}^{\prime}(C)=0,
$$

which has the advantage that we have at each iteration level a feasible solution for the state equation. We introduce the operator

$$
T(y) \stackrel{\text { def }}{=}\left[\begin{array}{c}
-e_{x}^{-1}(y) e_{C}(y) \\
I d_{\mathcal{C}}
\end{array}\right] .
$$


Then, for given Lagrange multipliers $(p, q)$ the reduced Hessian is defined by

$$
H(y ; p, q) \stackrel{\text { def }}{=} T^{*}(y) L_{y y}(y ; p, q) T(y)
$$

and it holds that

$H(y)=G_{\alpha, C C}(y)+e_{C}^{*}(y) e_{x}^{-*}(y)\left\{G_{\alpha, x x}(y)(\cdot, \cdot)+\left\langle e_{x x}(y)(\cdot, \cdot),(p, q)\right\rangle\right\} e_{x}^{-1}(y) e_{C}(y)$.

Then, the Newton algorithm reads as follows:

Let an admissible $C_{0}$ be given.

i) Set $k=0$ and $C^{0}=C_{0}$.

ii) Do while the stopping criterion is violated

(1) Set $y^{k}=\left(x\left(C^{k}\right), C^{k}\right)$ and $\left(p^{k}, q^{k}\right)=-e_{x}^{-*}\left(y^{k}\right) G_{\alpha, x}\left(y^{k}\right)$

(2) Solve $H\left(y^{k} ; p^{k}, q^{k}\right) \delta C^{k}=-\hat{G}_{\alpha}^{\prime}\left(C^{k}\right)$

(3) Set $C^{k+1}=C^{k}+\delta C^{k}, k=k+1$

iii) $C^{*} \stackrel{\text { def }}{=} C^{k}, y^{*} \stackrel{\text { def }}{=} y^{k}$, STOP.

Remark 1. We note that due to the structure of the reduced Hessian the Newton system in step ii)(2) has to be solved iteratively using e.g. a conjugate gradient method. Let us refer to this as the inner iteration. To provide the right hand side in ii) (2) one has to solve the nonlinear state system (1), (2) for $x^{k}=\left(V^{k}, \rho^{k}\right)$, and one needs to solve the adjoint system (5), (6) for $\left(p^{k}, q^{k}\right)$. These are all ingredients for the calculation of $\hat{G}_{\alpha}^{\prime}$ from (33).

Every application of $H\left(y^{k} ; p^{k}, q^{k}\right)$ in the $j$-th inner iteration amounts to two linear solves, namely

$$
v_{j}^{k}=e_{x}^{-1}\left(y^{k}\right) e_{C}\left(y^{k}\right) \delta C_{j}^{k}
$$

and

$$
w_{j}^{k}=e_{C}^{*}\left(y^{k}\right) e_{x}^{-*}\left(y^{k}\right)\left\{G_{\alpha, x x}\left(y^{k}\right)+\left\langle e_{x x}\left(y^{k}\right)\left(v_{j}^{k}, v_{j}^{k}\right),\left(p^{k}, q^{k}\right)\right\rangle\right\} .
$$

Let us come back to our previous numerical example to get more insight into the behavior of the algorithm. We tried to achieve an increase of the electron and hole current by $50 \%$ for the unsymmetric n-p-diode via optimal dopant profiling (for details we refer to [HP06]).

The conjugate gradient algorithm in the inner loop was terminated when the norm of the gradient became sufficiently small; to be more precise, in the $j$-th conjugate gradient step for the computation of the update in Newton step $k$ we stop if the residual $r_{j}^{k}$ satisfies

$$
\frac{\left\|r_{j}^{k}\right\|}{\left\|\hat{G}_{\alpha}^{\prime}\left(C^{0}\right)\right\|} \leq \min \left\{\left(\frac{\left\|\hat{G}_{\alpha}^{\prime}\left(C^{k}\right)\right\|}{\left\|\hat{G}_{\alpha}^{\prime}\left(C^{0}\right)\right\|}\right)^{q}, p \frac{\left\|\hat{G}_{\alpha}^{\prime}\left(C^{k}\right)\right\|}{\left\|\hat{G}_{\alpha}^{\prime}\left(C^{0}\right)\right\|}\right\} \quad \text { or } \quad j \geq 100 .
$$

Note, that $q \in(1,2)$ determines the convergence order of the outer Newton algorithm. The value of $p \in(0,1)$ is important for the first step of Newton's 


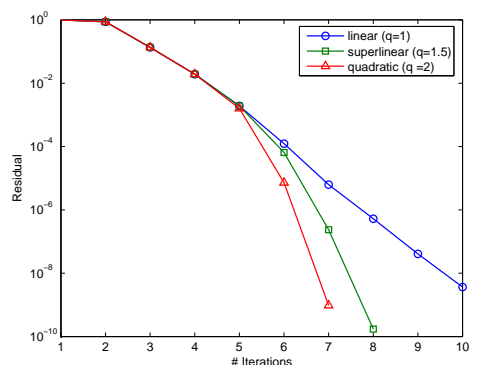

Fig. 3. Dependence of the residual on $q$

method, as for $k=0$ the norm quotients are all 1 ; for later steps, the influence of $q$ becomes increasingly dominant. In Figure 3 the decrease of the residual is depicted for different values of $q=1,1.5$, or 2 . As predicted by the general theory [Kel95] one gets linear, superlinear and quadratic convergence. Clearly, the parameter $q$ strongly influences the number of conjugate gradient steps, which can be seen from Figure 4 . While in the linear case $(q=1)$ we have an almost constant amount of CG steps in each each iteration, we get, as expected, a drastic increase towards the end of the iteration for the quadratic case $(q=2)$. Hence, the overall numerical effort in terms of CG steps is despite of the quadratic convergence much larger compared to the relaxed stopping criterion, which only yields linear convergence!

\subsection{Gummel Iterations}

Finally, we turn our attention to the minimization problem (10). Clearly, one can here also employ the previously discussed methods. But exploiting the special structure of the optimality system it is favorable to use a different iterative method in the spirit of the well-known Gummel iteration for the solution of the nonlinear state system [BP03]. Using a lower triangular approximation of the optimality system, we start with an potential $V$, and subsequently the continuity equations (2) with the given potential $V$ for $\rho$. With given potential $V$ and given $\rho$, we solve the adjoint equations (11), (12) to obtain the Lagrangian variables $p$ and $q$. Finally, we can perform a gradient step with respect to the design variable $V$ using the optimality equation (13). Due to the simple structure of this equation, it seems reasonable to discretize the Laplace term in an implicit way and thus, to solve

$$
-\beta\left(V_{x x}-V_{x x}^{*}\right)_{x x}+\tau\left(V_{x x}-V_{x x}^{*}\right)=\tau\left(V_{x x}^{o l d}-V_{x x}^{*}\right)-e^{V} u_{x} p_{x}+e^{-V} v_{x} q_{x},
$$

for an appropriately chosen damping parameter $\tau$. All together, we can write this iteration in the following form:

1. Choose an admissible $V^{0}$. 
2. For $k=1,2, \ldots$ solve consecutively

$$
\begin{aligned}
\partial_{x}\left(e^{V^{k}} \partial_{x} u^{k}\right) & =0 \\
\partial_{x}\left(e^{-V^{k}} \partial_{x} v^{k}\right) & =0 \\
\partial_{x}\left(e^{V^{k}} \partial_{x} p^{k}\right) & =0 \\
\partial_{x}\left(e^{-V^{k}} \partial_{x} q^{k}\right) & =0 \\
-\beta\left(V_{x x}^{k}-V_{x x}^{*}\right)_{x x}+\tau\left(V_{x x}^{k}-V_{x x}^{*}\right) & =\tau\left(V_{x x}^{k-1}-V_{x x}^{*}\right)-e^{V^{k}} u_{x}^{k} p_{x}^{k}+e^{-V^{k}} v_{x}^{k} q_{x}^{k} .
\end{aligned}
$$

The corresponding value of the doping profile can be computed independently by

$$
C^{k}-C^{*}=-\lambda^{2}\left(V_{x x}^{k}-V_{x x}^{*}\right)_{x x}+n^{k}-n^{*}-p^{k}+p^{*},
$$

where $n^{k}=e^{V^{k}} u^{k}$ and $p^{k}=e^{-V^{k}} v^{k}$.

Finally, we apply this algorithm to our numerical test case, where we want to have an increase of the overall current by $50 \%$. But now, we want to optimize a symmetric $\mathrm{n}-\mathrm{p}-$ diode.

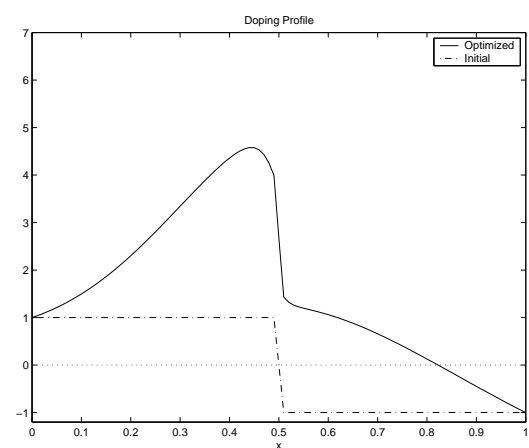

Fig. 5. Optimized Doping Profile

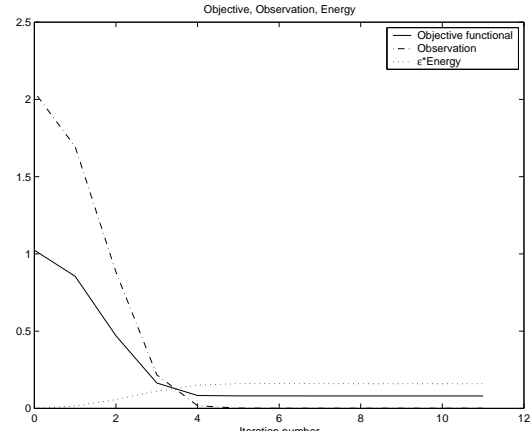

Fig. 6. Evolution of the Cost Functional

The optimal doping profile is depicted in Figure 5. But more interesting is the evolution of the cost function which is shown in Figure 6. The objective functional and the observation are reduced in a few iterations. Even if more iterations would be necessary, one needs to appreciate this Gummel-like algorithm, since its overall performance is again independent of the number of discrete design parameters and due its iterative structure it is a straightforward extension of the Gummel method. Hence, it is easy to incorporate into existing device simulation codes. Moreover, the numerical performance of this optimization algorithm is optimal, since we need in fact only two Gummel iterations for the solution of the minimization problem, i.e. the numerical complexity is double compared with a forward solve for the nonlinear state system. Note, that on the other hand we are here not feasible on each iteration level. 


\section{Acknowledgements}

This work was partially supported by the Johann Radon Institute for Computational and Applied Mathematics (Austrian Academy of Sciences ÖAW), by the Austrian National Science Foundation FWF through project SFB F 013 / 08, by the Sonderforschungsbereich 609 'Elektromagnetische Strömungsbeeinflussung in Matallurgie, Kristallzüchtung und Elektrochemie' , by the Sonderforschungsbereich 557 'Beeinflussung komplexer turbulenter Scherströmungen', both sponsored by the Deutsche Forschungsgemeinschaft, and by sponsored by the Forschungsschwerpunkt "Mathematik \& Praxis" (TU Kaiserslautern).

\section{References}

[BP03] Burger, M., Pinnau, R.: Fast optimal design of semiconductor devices, SIAM J. Appl. Math. 64, 108-126 (2003).

[GS92] Gajewski, H., Sommrey, J.: On the uniqueness of solutions of van Roosbroeck equations, ZAMM 72, 151-153 (1992).

[HP02a] Hinze, M., Pinnau, R.: Optimal control of the drift-diffusion model for semiconductor devices, in: Hoffmann, K.H., et. al. (eds.): Optimal Control of Complex Structures. Birkhäuser, Basel, Berlin (2002), 95-106.

[HP02b] Hinze, M., Pinnau, R.: An optimal control approach to semiconductor design, Math. Mod. Meth. Appl. Sci. 12, 89-107 (2002).

[HP05] Hinze, M., Pinnau, R.: Mathematical tools in optimal semiconductor design, to appear in TTSP (2005).

[HP06] Hinze, M., Pinnau, R.: A second order approach to optimal semiconductor design, to appear in JOTA (2006).

[JP01] A.Jüngel, Y.J.Peng, A model hierarchy for semiconductors and plasmas, Nonlin. Anal. 47 (2001), 1821-1832.

[Kel95] C.T. Kelley. Iterative Methods for Linear and Nonlinear Equations. SIAM, Philadelphia, 1995.

[MRS90] P.A.Markowich, C.A.Ringhofer, C.Schmeiser, SemiconductorEquations, Springer, Wien, New York, 1990.

[PSSS98] R.Plasun, M.Stockinger, R.Strasser, S.Selberherr, Simulation based optimization environment and its application to semiconductor devices, in: Proceedings IASTED Intl. Conf. on Applied Modelling and Simulation, 1998, pp. 313-316.

[St00] M. Stockinger, Optimization of ultra-low-power CMOS transistors, $\mathrm{PhD}$ Thesis, Technical University Vienna, 2000.

[Stea98] M.Stockinger, R.Strasser, R.Plasun, A.Wild, S.Selberherr, A Qualitative Study on Optimized MOSFET Doping Profiles, in: Proceedings SISPAD 98 Conf., Leuven, 1998, pp. 77-80. 